\title{
PERIODIC QUEUES IN HEAVY TRAFFIC
}

\author{
G. I. FALIN,* Moscow State University
}

\begin{abstract}
An analytic approach to the diffusion approximation in queueing due to Burman (1979) is applied to the $M(t) / G / 1 / \infty$ queueing system with periodic Poisson arrivals. We show that under heavy traffic the virtual waiting time process can be approximated by a certain Wiener process with reflecting barrier at 0 .
\end{abstract}

POISSON ARRIVALS; DIFFUSION APPROXIMATION

\section{Introduction}

An important property of periodic queues is the absence of a steady state in its usual sense. As a matter of fact, a special 'periodic stationary regime' exists. Consider for example the virtual waiting time process $W(t)$ in the $M(t) / G / 1 / \infty$ queue with FIFO discipline, Poisson arrival process with periodic intensity $\lambda_{t}$ (without loss of generality we can assume that the period equals 1) and general service time distribution function $B(x)$ with finite mean $\beta_{1}$ and variance $\sigma^{2}=\beta_{2}-\beta_{1}^{2}$. For example, $\lambda_{t}$ could be $\lambda+\beta \sin 2 \pi t$ with $\lambda>\beta \geqq 0$. If $\Lambda_{t}=\int_{0}^{t} \lambda_{u} d u$, $\lambda=\Lambda_{1}, \rho=\lambda \beta_{1}<1$, then there exists the family $H_{t}(x)$ of distribution functions, periodic functions of $t$ (i.e. $\left.H_{t+1}(x)=H_{t}(x)\right)$, such that $\lim _{t \rightarrow \infty}\left[P(W(t)<x)-H_{t}(x)\right]=0$. This fact is clearly seen from Figure 1 where the dependence of $E W(t)$ on $t$ is given in the case $\lambda_{t}=0 \cdot 5(1+\sin 2 \pi t)$, and $B(x)$ is the uniform distribution [0,2] (results were obtained using an Atari $130 \mathrm{XE}$ ). This 'periodic stationarity' imposes essential mathematical difficulties on the analysis of queues. Determination of the functions $H_{t}(x)$ and even determination of the mean value $\int_{0}^{1} d t \int_{0}^{\infty} x d H_{t}(x)$ can be made only with the help of a computer. To simplify the problem in practice, the 'principle of the mean' is usually used: a periodic queue is approximated by the corresponding stationary queue with arrival intensity $\lambda=$ $\lim _{t \rightarrow \infty} \frac{1}{t} \int_{0}^{t} \lambda_{u} d u=\Lambda_{1}$. Of course we have to find conditions when this approximation is reasonable.

In this note we shall show that this principle holds in heavy traffic in the sense of convergence of a scaled non-stationary process to a stationary process (which is in fact a reflected Brownian motion). To prove this we turn time heterogeneity into space heterogeneity (via a supplementary variable) which allows us to use the methodology of Burman [1].

\section{Main result}

Let us consider a sequence of $M(t) / G / 1 / \infty$ queues (indexed by a parameter $n$, although we shall usually omit it) with arrival rates $\lambda_{t}^{(n)}$. As a matter of convenience we suppose that the distribution of service time $B(x)$ does not depend on $n$.

Received 29 March 1988; revision received 27 January 1989.

* Postal address: Department of Probability, Mechanics and Mathematics Faculty, Moscow State University, Moscow 119899, USSR. 


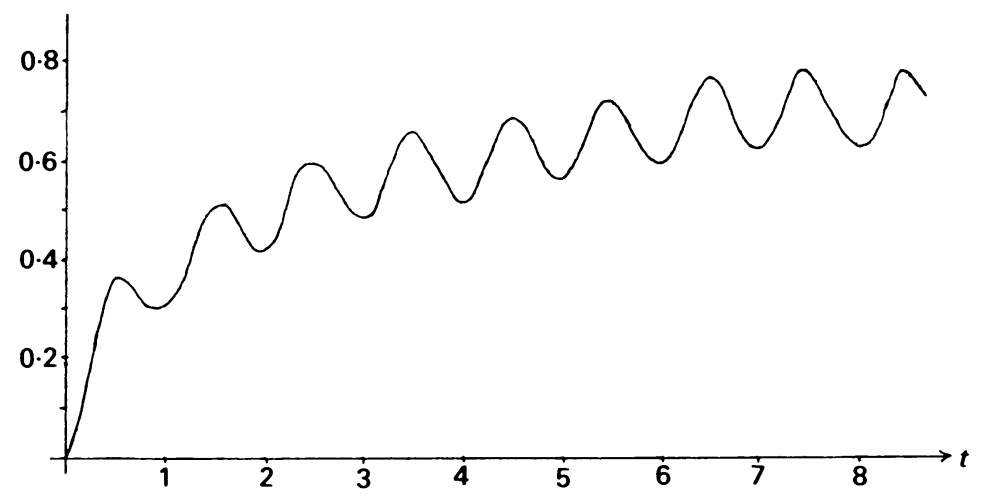

Figure 1

Theorem. If as $n \rightarrow \infty, \lambda^{(n)}=\int_{0}^{1} \lambda_{t}^{(n)} d t$ tends to $1 / \beta_{1}-0$ so that $\sqrt{n}\left(\rho^{(n)}-1\right)$, where $\rho^{(n)}=$ $\lambda^{(n)} \beta_{1}$, tends to $-m$, then the scaled processes $W(n t) / \sqrt{n}$ converge (in the sense of convergence of finite-dimensional distributions) to a Brownian motion with reflecting barrier at the origin with infinitesimal mean $m$ and infinitesimal variance $\beta_{2} /\left(2 \beta_{1}\right)$.

Proof. The main idea of the proof consists in turning time heterogeneity into space heterogeneity. To do this let us denote by $\tau(t)$ the fractional part of $t$. The process $(W(t), \tau(t))$ is a time-homogeneous Markov process with state space $R_{+} \times[0,1]$, but with transition characteristics which depend on the second coordinate $\tau$ of a point $(x, \tau)$ of the state space.

Another problem now arises. Under heavy traffic only the first coordinate of the process $(W(t), \tau(t))$ will converge to a diffusion process. But this difficulty can be removed by using the method of proving functional limit theorems in queueing theory due to Burman [1].

The infinitesimal generator of the process $(W(t), \tau(t))$ is expressed as follows:

$$
\begin{gathered}
A f(x, \tau)=\lambda_{\tau} \int_{0}^{\infty}[f(x+u, \tau)-f(x, \tau)] d B(u)+f_{\tau}^{\prime}(x, \tau)-f_{x}^{\prime}(x, \tau), \quad \text { if } \quad x>0, \quad 0<\tau<1, \\
A f(0, \tau)=\lambda_{\tau} \int_{0}^{\infty}[f(u, \tau)-f(0, \tau)] d B(u)+f_{\tau}^{\prime}(0, \tau), \quad \text { if } \quad 0<\tau<1,
\end{gathered}
$$

and operates on functions $f(x, \tau)$ satisfying the boundary condition $f(x, 1)=f(x, 0)$.

For the generator $A_{n}$ of the scaled process $(W(n t) / \sqrt{n}, \tau(n t))$ these formulas become:

$$
\begin{aligned}
A_{n} f(x, \tau) & =n \lambda_{\tau} \int_{0}^{\infty}\left[f\left(x+\frac{1}{\sqrt{n}} u, \tau\right)-f(x, \tau)\right] d \tilde{B}(u)+n f_{\tau}^{\prime}(x, \tau)-\sqrt{n} f_{x}^{\prime}(x, \tau) \\
& =\sqrt{n} \lambda_{\tau} f_{x}^{\prime}(x, \tau) \beta_{1}+\frac{1}{2} \lambda_{\tau} \beta_{2} f_{x x}^{\prime \prime}(x, \tau)+n f_{\tau}^{\prime}(x, \tau)-\sqrt{n} f_{x}^{\prime}(x, \tau)+o(1),
\end{aligned}
$$

if $x>0,0<\tau<1$; and

$$
\begin{array}{rl}
A_{n} f(0, \tau)=n \lambda_{\tau} \int_{0}^{\infty}\left[f\left(\frac{u}{\sqrt{n}}, \tau\right)-f(0, \tau)\right] d & B(u)+n f_{\tau}^{\prime}(0, \tau) \\
& =\sqrt{n} \lambda_{\tau} f_{x}^{\prime}(0, \tau) \beta_{1}+\frac{1}{2} \lambda_{\tau} \beta_{2} f_{x x}^{\prime \prime}(0, \tau)+n f_{\tau}^{\prime}(0, \tau) .
\end{array}
$$

The boundary condition does not change. 
For any twice continuously differentiable function $f(x)$ define

$$
f_{n}(x, \tau)=f(x)+\frac{1}{\sqrt{n}} f^{\prime}(x) g(\tau)+\frac{1}{n} f^{\prime \prime}(x) h(\tau),
$$

where the functions $g(\tau), h(\tau)$ will be defined below. For such functions we have;

$$
\begin{gathered}
A_{n} f_{n}(x, \tau)=\sqrt{n} f^{\prime}(x)\left[\lambda_{\tau} \beta_{1}-1+g^{\prime}(\tau)\right]+f^{\prime \prime}(x)\left[\left(\lambda_{\tau} \beta_{1}-1\right) g(\tau)+\frac{1}{2} \lambda_{\tau} \beta_{2}+h^{\prime}(\tau)\right]+o(1), \\
A_{n} f_{n}(0, \tau)=\sqrt{n} f^{\prime}(0)\left[\lambda_{\tau} \beta_{1}+g^{\prime}(\tau)\right]+f^{\prime \prime}(0)\left[\lambda_{\tau} \beta_{1} g(\tau)+\frac{1}{2} \lambda_{\tau} \beta_{2}+h^{\prime}(\tau)\right]+o(1) .
\end{gathered}
$$

The boundary condition becomes;

$$
g(1)=g(0), \quad h(1)=h(0) .
$$

From the above it is easy to see that $A_{n} f_{n}$ can converge to a limit which does not depend on $\tau$ only if the functions $g(\tau), h(\tau)$ satisfy the equations:

$$
\lambda_{\tau} \beta_{1}-1+g^{\prime}(\tau)=\frac{1}{\sqrt{n}} c_{1}, \quad\left(\lambda_{\tau} \beta_{1}-1\right) g(\tau)+\frac{\lambda_{\tau} \beta_{2}}{2}+h^{\prime}(\tau)=c_{2},
$$

where $c_{1}, c_{2}$ are to be determined from the boundary conditions.

The first equation yields

$$
g(\tau)=\left(\tau-\Lambda_{\tau} \beta_{1}\right)+\frac{1}{\sqrt{n}} c_{1} \tau+g(0) .
$$

By the boundary condition $g(1)=g(0)$ we get

$$
c_{1}=-\sqrt{n}\left(1-\rho^{(n)}\right)=-m+o(1),
$$

so $g(\tau)=\rho \tau-\Lambda_{\tau} \beta_{1}+g(0)$. This allows us to obtain

$$
h(\tau)=c_{2} \tau+\frac{1}{2} \Lambda_{\tau} \beta_{2}+\int_{0}^{t}\left(1-\lambda_{u} \beta_{1}\right) g(u) d u+h(0) .
$$

The boundary condition $h(1)=h(0)$ gives

$$
C_{2}=\beta_{2} /\left(2 \beta_{1}\right)+o(1),
$$

Using the functions $g(\tau), h(\tau)$ we get

$$
\lim _{n \rightarrow \infty} A_{n} f_{n}(x, \tau)=-m f^{\prime}(x)+\frac{\beta_{2}}{2 \beta_{1}} f^{\prime \prime}(x)=A_{0} f(x) .
$$

The generator $A_{0}$ corresponds to a diffusion process with infinitesimal mean $m$ and infinitesimal variance $\beta_{2} /\left(2 \beta_{1}\right)$.

Similar analysis of behaviour at the boundary $x=0$ implies that functions $f(x)$ from the domain of the generator $A_{0}$ have to satisfy the condition $f(0)=0$. This means that the generator $A_{0}$ corresponds to diffusion with a reflecting barrier at the origin.

To complete the proof it is sufficient to refer to results in [1] and [2] which imply that the above allow us to guarantee the desired convergence.

\section{References}

[1] Burman, D. Y. (1979) An Analytic Approach to Diffusion Approximation in Queueing. Ph.D. Thesis, Department of Applied Mathematics, Courant Instutute of Mathematics, New York University.

[2] ETHIER, S. AND KURTZ, T. (1986) Markov Processes: Characterization and Convergence. Wiley, New York. 